\title{
A Positional Pitfall in the Interpretation of Chest Radiogram in a Newborn
}

\author{
Jana Lozar Krivec ${ }^{1}$, Damjana Ključevšek ${ }^{2}$, Mojca Glušič ${ }^{2}$
}

${ }^{1}$ Division of Paediatrics, Department of Neonatology, University Medical Centre Ljubljana, Slovenia, ${ }^{2}$ Division of Paediatrics, Department of Radiology, University Medical Centre Ljubljana, Slovenia
Received: May 22, 2019

Accepted: August 9, 2019

Key Words: Sternal Ossification Centres • Round Nodules • Newborn - Chest X-Ray • Interpretation • Portable X-Ray Unit.
A three-day-old term newborn was admitted to the neonatal unit due to tachypnoea and mild cyanosis. Physical examination revealed tachypnoea and mild chest retractions, the precordial pulsations were exaggerated, and the second heart sound was narrowly split and accentuated. Haematological and biochemical laboratory values were normal. Chest X-ray was performed with a portable $\mathrm{X}$-ray unit and analysed by a resident. During the exposure the newborn was in an inappropriate position, the baby's chest was rotated on the radiogram and the left arm was projected over the lower left lung. The resident reported six round clearly demarcated nodules (arrows) of unknown aetiology projecting into the right lung (Fig. 1). The remaining chest structures were within normal limits. On the next day, echocardiogram revealed an elevated pulmonary resistance, a small atrial septal defect and patent ductus arteriosus, which explained the infant's condition. The chest $\mathrm{X}$-ray was reviewed by

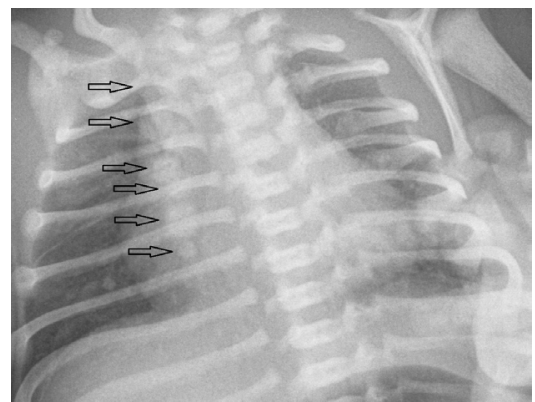

Fig. 1.

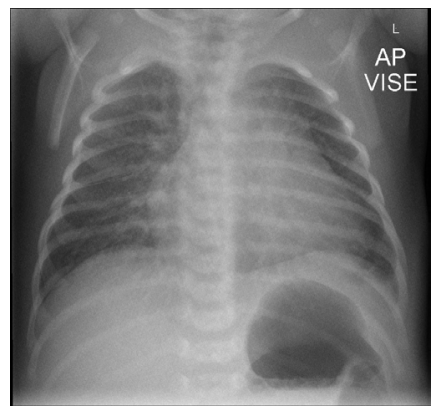

Fig. 2a.

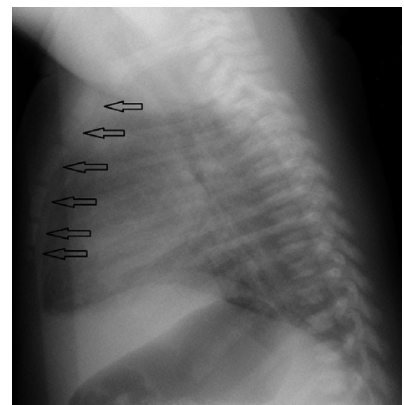

Fig. 2 b. 
a paediatric radiologist who concluded that the 'nodules' were sternal ossification centres. On the follow-up chest X-ray (Fig. 2a and $2 \mathrm{~b}$ ) of the baby in a fixed upright position (immobilisation by "baby-fix"), on the anteroposterior view the 'nodules' were no longer seen, while sternal ossification centres (arrows) were shown on the lateral view. Inappropriate chest X-ray position can make normal structures such as the sternal ossification centres visible, which were in this case misinterpreted as pathological structures by a resident. Therefore, for quality report of chest
X-ray, education on positional and technical factors influencing the quality of X-ray images and their evaluation is necessary before commencing interpretation of different chest structures.

Authors' Contributions: Conception and design: JLK; Acquisition, analysis and interpretation of data: JLK, DK, and MG; Drafting the article: JLK; Revising the article critically for intellectual content: JLK, DK, and MG; Approved final version of the manuscript: JLK, DK and MG.

Conflict of Interest: The authors declare that they have no conflict of interest. 